\title{
INFILTRACIÓN DEL NERVIO PUDENDO GUIADA POR TOMOGRAFÍA AXIAL COMPUTADA, POR VÍA TRANSGLÚTEA: TERAPIA FRENTE AL DOLOR OCASIONADO POR NEURALGIA DEL NERVIO PUDENDO
}

\author{
Paolo Ricci A. ${ }^{1}$, Rodrigo Lema C. ${ }^{1}$, Vicente Solà D. ${ }^{1}$, Alex Wash F. ${ }^{2}$, Jack Pardo S. ${ }^{1}$ \\ 1 Unidad de Ginecología, Departamento de Obstetricia y Ginecología, ${ }^{2}$ Departamento de Radiología, \\ Clínica Las Condes.
}

\section{RESUMEN}

Antecedentes: La neuralgia del nervio pudendo pocas veces es sospechada y menos diagnosticada. Por esta razón, las pacientes que la padecen, consultan múltiples veces antes de llegar a un diagnóstico definitivo. Objetivo: Revisar la seguridad y eficacia de la infiltración de nervio pudendo, en el tratamiento del dolor en pacientes con neuralgia del nervio pudendo. Método: Seguimiento prospectivo de cinco pacientes ingresadas bajo el diagnóstico de síndrome de atrapamiento del nervio pudendo. La edad media de las pacientes fue 45 años. Debían tener 2 criterios mayores o 1 criterio mayor asociado a 2 criterios menores. Dolor con al menos 6 meses de evolución. Sin tratamientos previos. A todas se les realizó encuesta de síntomas y signos de atrapamiento del nervio pudendo. Todas fueron infiltradas con corticoides y anestésico, guiada por tomografía axial computada. Se comparó el dolor antes y después de la infiltración con escala de 0 a 10. Resultados: No hubo complicaciones durante el procedimiento. Todas disminuyeron el dolor después de la infiltración, y señalaron estar conformes con los resultados. Conclusión: La infiltración del nervio pudendo guiada por tomografía axial computada, es una técnica segura y eficaz, en el control o disminución del dolor pelviano, ocasionado por neuralgia del nervio pudendo. Sólo el seguimiento permitirá evidenciar la mantención de los buenos resultados, o la necesidad de repetir la infiltración o de realizar cirugía de descompresión.

\section{PALABRAS CLAVE: Nervio pudendo, neuralgia del nervio pudendo, dolor pélvico crónico, infiltración de nervio pudendo}

\section{SUMMARY}

Background: The pudendal neuralgia infrequently is suspected and diagnosed. Therefore the patients who suffer it consult manifold times before arriving at a definitive diagnosis. Objective: To review the security and effectiveness of the pudendal nerve infiltration in the pudendal neuralgia treatment. Method: Prospective study of five patients under entrapment pudendal nerve syndrome diagnosis. The media age was 45 years old. All patients must have 2 greater criteria or 1 greater criteria associate to 2 smaller criteria. The pain must have an evolution of at least 6 months. They must not have antecedent of infiltration or surgery like treatment of this syndrome. A questionnaire of entrapment pudendal nerve syndrome was applied. The infiltration was with corticoids and anesthetic guided by CT scan. The patients classified their pain with a subjective scale; in which 0 is the pain absence and 10 is the maximum pain. The pain was compared before and after infiltration. Results: Complication did not appear during the infiltration procedure. In one patient an 
asymmetry in the spine corresponding to the side of pain was observed. All patients diminished the pain after the infiltration. All indicated to be in agreement with the results. Conclusion: The pudendal nerve infiltration guided by CT scan is safe and effective technique in the treatment or diminish of the pudendal neuralgia. Only the follow-up will allow demonstrating the good results, or the necessity to repeat the infiltration, or the necessity of decompression surgery.

\section{KEY WORDS: Pudendal nerve, pudendal neuralgia, pelvic chronic pain, pudendal nerve infiltration}

\section{INTRODUCCIÓN}

La neuralgia del nervio pudendo (NP) puede ocasionarse por compromiso inflamatorio perineural, lo que causa una verdadera compresión extrínseca. Teóricamente, cualquier alteración que afecte los tejidos alrededor del nervio, en cualquier parte de su trayecto, pueden llevar a una neuralgia pudenda. Sin embargo, existen territorios que con mayor frecuencia pueden afectarse. Entre estos, se encuentra la pinza que se produce entre el ligamento sacrotuberoso y el sacroespinoso, al igual que otros "síndromes de túnel", en que se comprimen los nervios en su trayecto, al pasar entre fascias. También el NP puede comprimirse en su porción al interior del canal de Alcock. Por estas razones la neuralgia del pudendo, puede ser ocasionada por lo que se conoce genéricamente como síndrome de atrapamiento del nervio pudendo, o más específicamente en el caso antes mencionado, como síndrome del canal de Alcock.

El síndrome de atrapamiento del nervio pudendo fue descrito por primera vez en 1987 por Amarenco y cols (1). El motivo principal de consulta de estos pacientes, es el dolor perineal y/o pelviano, que puede asociarse a disfunción urinaria, anal e incluso sexual (2). Se trata de una patología poco conocida y por ello raramente diagnosticada. Por estos motivos, las pacientes que padecen este síndrome, frecuentemente, consultan a múltiples médicos antes de llegar a un diagnóstico definitivo. El dolor puede ser tan intenso y agobiante que se han descrito suicidios por esta razón.

Actualmente se desconoce la incidencia y prevalencia de esta patología. Sin embargo, se estima que en Europa y Estados Unidos, 1 de cada 7 mujeres padecerá en alguna etapa de su vida esta alteración, en forma transitoria o crónica (2). Entre las causas o factores desencadenantes del síndrome de atrapamiento del nervio pudendo, se encuentra: el trauma, parto vaginal y episiotomía, cirugía ginecológica, radioterapia pelviana, defectos congénitos, algunas profesiones y prácticas deportivas $(1,2)$.
El tratamiento de esta patología es secuencial, ya que comprende en una primera etapa medidas de autocuidado y la infiltración del NP. El autocuidado consiste en adoptar medidas de protección perineal. Para ello es necesario dejar las actividades que desencadenan o aumentan el dolor, como por ejemplo el ciclismo. Además se puede utilizar una plataforma perineal, que permite que la presión provocada por el peso corporal al sentarse, se realice sobre las tuberosidades isquiáticas, disminuyendo la compresión sobre el nervio en su trayecto.

La infiltración del NP por vía transglútea, permite inyectar corticoides y anestésicos, que disminuirán el dolor y la inflamación. La respuesta no siempre es completa, sin embargo, el dolor puede llegar a disminuir en forma importante. Según la respuesta, se puede repetir cada 4 a 6 semanas, con un máximo de 6 infiltraciones.

El objetivo de esta comunicación es presentar un seguimiento prospectivo, de una serie de pacientes con síndrome de atrapamiento del nervio pudendo, tratados por medio de infiltración con corticoides y anestésicos, por vía transglútea, bajo visión de tomografía axial computada.

\section{MATERIAL Y MÉTODO}

Se realizó un seguimiento prospectivo, en cinco pacientes ingresadas bajo el diagnóstico de Síndrome de Atrapamiento del Nervio Pudendo, a la Unidad de Ginecología del Departamento de Obstetricia y Ginecología de Clínica Las Condes. Entre abril de 2007 y abril de 2008. Los criterios de inclusión fueron:

1.-Diagnóstico del síndrome de atrapamiento del nervio pudendo.

Se utilizó la certificación de 2 criterios mayores o 1 criterio mayor asociado a 2 criterios menores.

Criterio mayor:

- Área dolorosa en el trayecto del nervio o sus ramas:

a) nervio rectal inferior: ano, perineo, tercio inferior del recto, nalgas.

b) nervio perineal: labios menores, labios mayores, tercio inferior de la vagina, periuretra. 
c) nervio dorsal del clítoris: área cutánea del clítoris y sínfisis pubiana.

- El dolor se reproduce al comprimir el tronco pudendo (signo de Tinel) (3).

Criterio menor:

- Sensación de dolor neuropático.

- Existencia de una posición que produce dolor y/o una que alivia (anti-álgica), por ejemplo: el dolor aumenta al sentarse y se alivia en decúbito.

- Identificación de un factor etiológico o desencadenante, por ejemplo: aparición del dolor después de un parto, traumatismo perineal, cirugía perineal, etc.

- Ausencia de otra causa que explique el dolor en el área pélvica (patología ginecológica como endometriosis, etc.).

2.-Sin tratamiento previo con infiltración o quirúrgico, por este motivo. Se acepta sólo tratamiento sintomático previo con analgesia y/o anti-inflamatorios vía oral.

3.-Dolor pélvico y/o perineal al menos por 6 meses de evolución, con escasa o nula respuesta frente a terapia analgésica y/o anti-inflamatoria vía oral.

A todas las pacientes ingresadas se les aplicó el cuestionario de Síndrome de Atrapamiento del Nervio Pudendo:

1.¿Cuánto tiempo tiene el dolor?

2.¿Hay algún evento que coincidió con el inicio del dolor?
3. ¿Cuánto duele en una escala de 0 a 10 ?

4.¿El dolor es: "quemante", "tirante", "aplastante", "cortante", otros (señalar)?

5.¿Presencia de dolor en la región de las nalgas?

6. ¿Presencia de dolor vaginal?

7.¿Presencia de dolor vulvar?

8. ¿El dolor es peor al sentarse?

9. ¿Sentarse en el baño no es tan doloroso como sentarse en una superficie plana?

10. ¿El dolor es peor al estar de pie?

11. ¿El dolor es peor al acostarse?

12. ¿El dolor es peor al acostarse hacia un lado que al otro?

13. ¿Hay dolor con la defecación?

14. ¿Hay dolor con la relación sexual?

15. ¿Hay dolor al orinar?

16. ¿Tengo problemas para controlar mi vejiga?

17. ¿Tengo problemas para controlar mi intestino?

18. ¿Tengo problemas para controlar la expulsión de gases?

A todas se les realizó un Rolling Test (4), tabulando su resultado. Esta prueba consiste en desplazar con fuerza la piel y el celular subcutáneo, sobre el trayecto de las ramas del nervio pudendo. Al realizar esta maniobra, se reproduce el dolor en la zona inervada.

Las pacientes ingresadas al estudio tenían entre 41 y 54 años, con una media de 45 años (Tabla I).

Tabla I

PACIENTES INGRESADAS AL ESTUDIO

\begin{tabular}{ccccccc}
\hline Paciente & $\begin{array}{c}\text { Edad } \\
\text { (años) }\end{array}$ & $\begin{array}{c}\text { Tiempo dolor } \\
\text { (meses) }\end{array}$ & $\begin{array}{c}\text { Tipo } \\
\text { dolor }\end{array}$ & $\begin{array}{c}\text { Consultas } \\
\text { previas }\end{array}$ & $\begin{array}{c}\text { Rolling } \\
\text { test }\end{array}$ & $\begin{array}{c}\text { Intensidad } \\
\text { dolor }\end{array}$ \\
\hline 1 & 45 & 16 & U,P,C & 5 & Positivo & 8 \\
2 & 42 & 12 & U,P & 10 & Positivo & 7 \\
3 & 54 & 9 & U,C & 6 & Negativo & Positivo \\
4 & 49 & 10 & U,P & 7 & Negativo & 8 \\
\hline
\end{tabular}

Tipo de dolor: U (urente), P (presión), C (cortante).

Intensidad del dolor, medida por escala subjetiva entre 0 y 10 . Donde 0 es sin dolor y 10 la máxima intensidad. Número asignado por el propio paciente. 
Técnica de infiltración del NP bajo visión guiada por $T A C$ : Con la paciente en posición decúbito ventral, en la camilla de TAC, se realizó marcación de la piel glútea, por medio de la adherencia de una grilla (Figura 1). Luego se realiza una tomografía de la región pélvica. La imagen de la grilla sirve como referencia para escoger el mejor lugar para introducir la aguja a través de la piel (Figura 2). Se marca con lápiz en la piel de la zona escogida y se retira la griIla. Se realiza una pápula con lidocaína y en parte del trayecto en el músculo (Figura 3). Se introduce

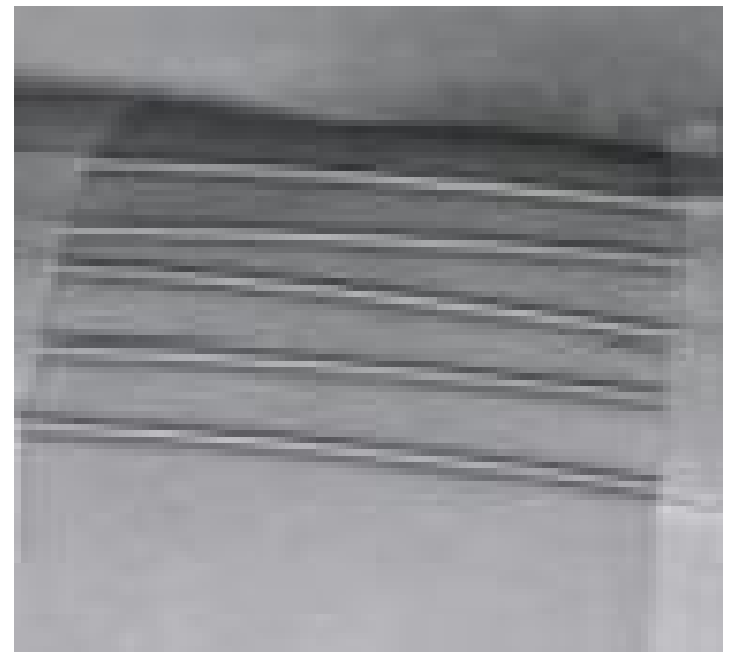

Figura 1. Grilla utilizada en la piel de la zona glútea, como guía para marcar el ingreso de la aguja de infiltración. La grilla permite tener puntos de referencia en la imagen obtenida por medio de la tomografía axial computada.

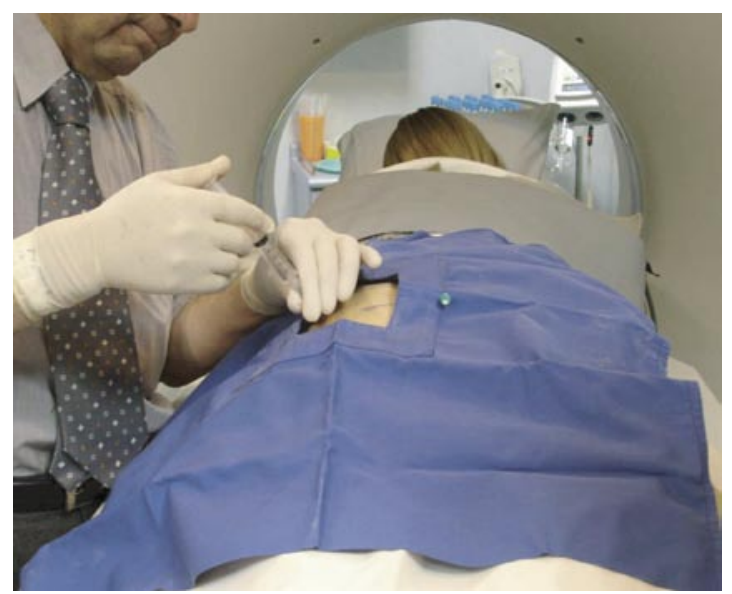

Figura 3. Marcada la piel en la zona de referencia para ingreso de la aguja, se infiltra con anestesia, por medio de una pápula subdérmica y parte del trayecto que recorrerá la aguja de infiltración en el músculo. un catéter a través de la zona marcada. Se inyecta una pequeña cantidad de medio de contraste para mejorar la visión. Se realiza nuevamente observación a través de tomografía, para identificar y corregir la posición de las agujas a ambos lados (Figura 4). Estas deben quedar con sus extremos en posición adyacente a ambas espinas ciáticas. Una vez que se tiene la seguridad que es la mejor posición de las agujas, se inyecta $80 \mathrm{mg}$ de metilprednisoIona acetato y bupivacaina clorhidrato $0,5 \%$ (diluida al 50\%), a ambos lados (Figura 5).

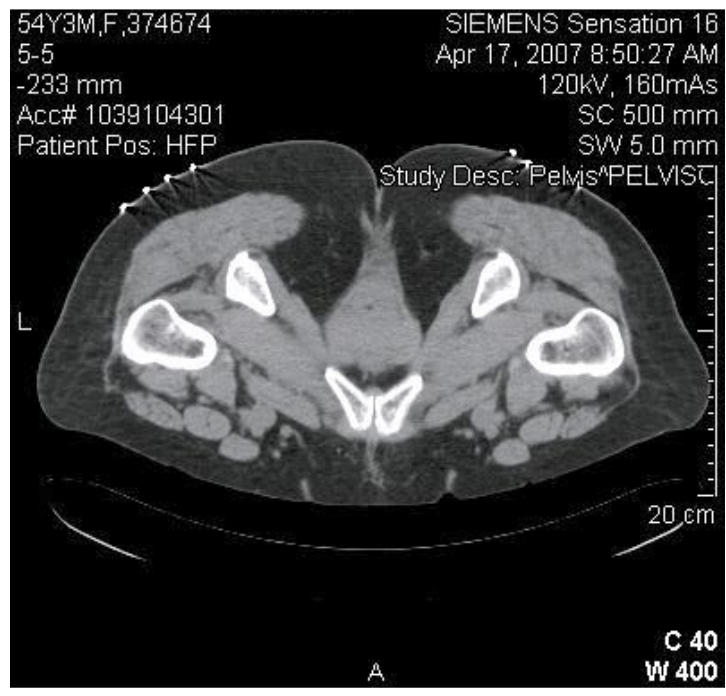

Figura 2. Imagen de la pelvis en la tomografía axial computada. Se observa la grilla como punto de referencia para la inserción de las agujas.

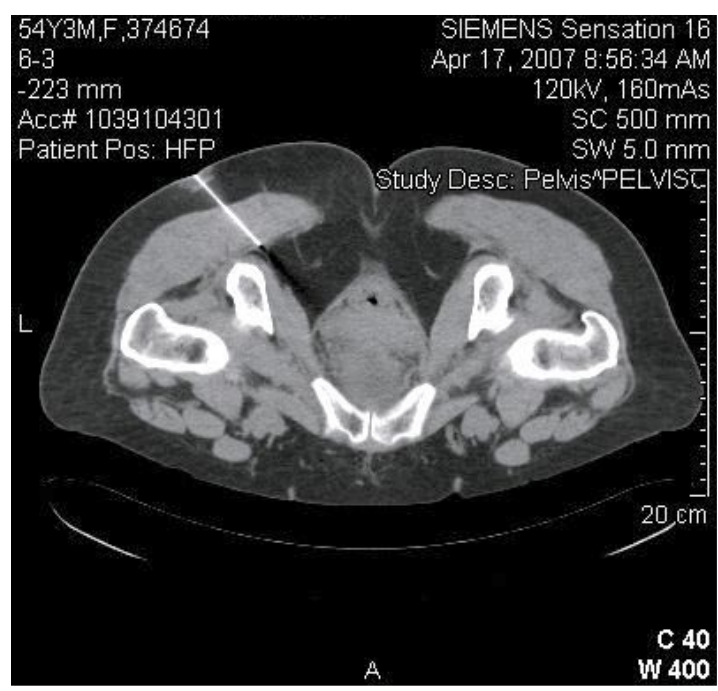

Figura 4. Inserción de la aguja con medio de contraste. Se revisa su correcta posición, por medio de la tomografía. 


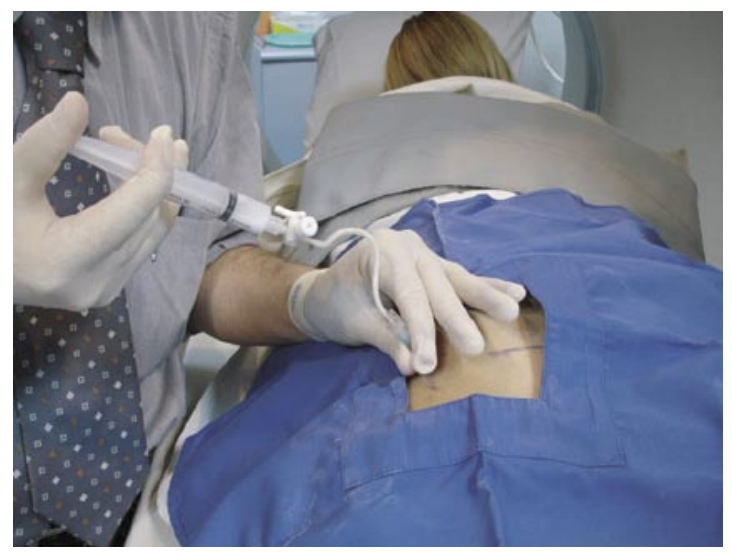

Figura 5. Verificada la posición correcta de la aguja, se inyecta el corticoide y el anestésico.

Seguimiento y medición de la intensidad del dolor de las pacientes:

-Al ingreso se preguntó por la intensidad del dolor, para lo cual se utilizó una escala subjetiva en la que cada paciente se clasificó a si misma. La escala comprendía desde 0 a 10, donde 0 es ausencia de dolor y 10 es la máxima intensidad.

-Se realizó control a los 7, 30 y 60 días.

-Se preguntó durante los controles por la evolución e intensidad del dolor.

\section{RESULTADOS}

La media de consultas a médicos, previamente al diagnóstico y tratamiento, fue de 6 , con un rango de 5 a 10. A todas se les había recetado y utilizaban analgésicos y/o anti-inflamatorios. Todas utilizaban analgésicos neuropáticos (tipo gabapentina).

Presentaban el dolor entre 8 y 16 meses, con una media de 10. La característica del dolor, descrita con mayor frecuencia, fue el urente o quemante y el de tipo presión. Respecto a la intensidad subjetiva del dolor, al ingreso señalaron que era entre $7 y$ 8 , con una media de 8 . Tres de las pacientes señalaron haber llegado hasta 10 en algunos episodios. Los otros resultados del cuestionario se presentan en la Tabla II.

No se registraron complicaciones en el procedimiento de infiltración. El dolor después del procedimiento en todas disminuyó. Registrándose en la escala subjetiva del dolor, a los 7 días, rangos entre 2 y 5 , con una media de 4 (Tabla III). Al preguntarles por la conformidad del resultado, todas señalaron que estaban muy conformes.

\section{DISCUSIÓN}

La neuralgia del NP es un síndrome que raramente se reconoce y diagnostica, por lo que en la mayoría de las veces no se trata correctamente (5). Los signos y síntomas del la neuralgia del NP, son muchos y de gran variabilidad individual (6). Sin embargo, el principal motivo de consulta es el dolor, el cual puede ser de tal magnitud, que afecta la calidad de vida de quien lo padece. Generalmente se describe un dolor perineal tipo quemante o urente, tal como se registró en nuestra serie $(7,8)$.

Entre los exámenes que se pueden utilizar para objetivar un compromiso del NP, se encuentran las pruebas neurofisiológicas como la electromiografía y la medición de latencia de las terminaciones nerviosas (4). Sin embargo, la medición de la conducción nerviosa no siempre se encuentra alterada, por lo que debemos guiarnos preferentemente por la clínica (4,9-11). Es muy probable que esta alteración sea proporcional al grado de compromiso del nervio.

El tratamiento del dolor provocado por compromiso del NP, debe incluir medidas de autocuidado. Entre estas, el uso de plataformas perineales y evitar posturas o actividades que desencadenen el dolor. Entre las actividades que frecuentemente pueden desencadenar o agravar este síndrome, se encuentra el ciclismo. Actualmente se encuentran disponibles sillines especiales, que permiten dar una protección adicional, disminuyendo la posibilidad de compresión del nervio.

Se considera que las terapias de infiltración pueden ser beneficiosas hasta en la mitad de las pacientes, mientras que las cirugías de descompresión solo en un tercio (5). La infiltración, contempla un tratamiento de menor invasión, respecto a la descompresión, que implica una cirugía en un territorio anatómico complejo. Terapias futuras, tal vez, podrían considerar la neuromodulación, lo que no ha sido explorado adecuadamente aún.

Una experiencia similar a la nuestra, presentada por Amarenco y cols (3), demostró un resultado satisfactorio en el control del dolor, en 9 de 15 pacientes, tratados con infiltración bajo visión, guiada por tomografía. 
Tabla II

RESPUESTAS A CUESTIONARIO DE SÍNDROME DE ATRAPAMIENTO DEL NERVIO PUDENDO

\begin{tabular}{|c|c|c|c|c|}
\hline Paciente 1 & Paciente 2 & Paciente 3 & Paciente 4 & Paciente 5 \\
\hline \multicolumn{5}{|c|}{ ¿Cuánto tiempo tiene el dolor? } \\
\hline 16 meses & 17 meses & 9 meses & 10 meses & 8 meses \\
\hline
\end{tabular}

¿Hay algún evento que coincidió con el inicio del dolor?

NO Sí No No Ní

¿Cuánto duele en una escala de 0 a $10 ?$

8

7

8

8

¿El dolor es: "quemante", "tirante","aplastante", "presión", "cortante", otros (señalar)
$U, P, C$
$\mathrm{U}, \mathrm{P}$
$\mathrm{U}, \mathrm{C}$
$U, P$
U,P,C

¿Tengo dolor en la región de las nalgas?

sí sí sí sí sí

¿Tengo dolor en la vagina?

sí sí sí sí sí

¿Tengo dolor en los labios de la vulva?

sí Sí No sí

¿El dolor es peor al sentarse?

sí Sí sí Sí Sí

¿Sentarse en el baño no es tan doloroso como sentarse en una superficie plana?

sí sí sí sí sí

¿El dolor es peor al estar de pie?

NO NO NO N NO N

¿El dolor es peor al acostarse?

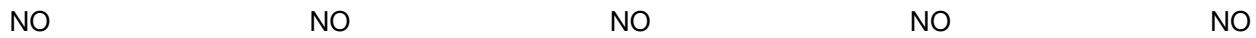

¿El dolor es peor al acostarse hacia un lado respecto del otro?
sí
sí
sí
NO
sí

¿Hay dolor con la defecación?

sí Sí $\quad$ sí $\quad$ NO Sí

¿Hay dolor con la relación sexual?
sí
Sí
sí
Sí
sí

¿Hay dolor al orinar?

NO

NO

NO

NO

NO

¿Tengo problemas para controlar mi vejiga?
NO
NO
NO
NO
NO

¿Tengo problemas para controlar mi intestino?

NO

NO

NO

NO

NO

¿Tengo problemas para controlar la expulsión de gases?

NO

NO

NO

NO

NO 
Respecto a la razón de la eficiencia de este tratamiento, se basa en que permite inyectar un corticoide y un anestésico, que disminuyen la inflamación de los tejidos perineurales, que en muchas ocasiones es lo que produce una compresión y atrapamiento del nervio. Esta inflamación puede desencadenarse por cirugías previas, trauma o actividades deportivas que significan un continuo trauma en la zona. Sin embargo, a veces existen defectos anatómicos que desencadenan o permiten que se mantenga la inflamación en el tiempo.

Un buen lugar para infiltrar, es a nivel de las espinas, lugar en relación al nervio que frecuentemente se ve afectado. Lo que además, permite disminuir una posible inflamación y compresión a nivel del canal de Alcock. En otros casos la afección del nervio, puede ser por un atrapamiento a nivel de las pinzas, a nivel de los ligamentos sacrotuberoso y sacroespinal o la fascia del músculo obturador interno (12-14). En casos en que hemos detectado dolor localizado a estos niveles, hemos utilizado infiltraciones por vía vaginal, con respuesta satisfactoria, en pacientes que se encuentran en seguimiento actualmente.

No debemos olvidar, que las tres ramas terminales del NP, poseen en diferentes proporciones, fibras motoras, sensitivas y autonómicas. Por ello, la alteración de este nervio puede causar signos y síntomas diversos. Se estima que un $30 \%$ es autonómico y el $70 \%$ es somático (50\% sensitivo y $20 \%$ motor). Por estas razones, podemos observar en casos crónicos, alteraciones de la piel (2), incontinencia de orina (15) o anal (16). En las pacientes de nuestra serie se presentaron cambios en la piel sólo en un caso. No se observó incontinencia urinaria ni fecal, seguramente por no contar con un compromiso de mayor tiempo.

En los casos en que la respuesta no es adecuada por no reducir significativamente el dolor, será necesario considerar la repetición de la infiltración. Sin embargo, aquellos casos en que no logran definitivamente una buena respuesta, deberán considerar una posible cirugía. Esta puede realizarse por vía transperineal, transglútea o transvaginal (16-17).

Entre los medicamentos de apoyo para aliviar el dolor asociado a este síndrome, como los analgésicos neuropáticos, tipo gabapentina y sobretodo la asociación a amitriptilina, presentan muy buenos efectos en el control y mantención de estos pacientes, en los que se ha realizado la primera infiltración. Estas pacientes, se encuentran en protocolos de seguimiento para registrar la evolución y resultados de nuevas infiltraciones.

\section{CONCLUSIONES}

La infiltración profunda bajo visión TAC, en pacientes que cumplen con los criterios para el diagnóstico de síndrome de atrapamiento del nervio pudendo, es un procedimiento seguro y confiable. Ya que permite reducir el dolor a rangos soportables, que mejoran la calidad de vida de estas pacientes.

Sólo la observación y control en el tiempo, permitirán determinar la mantención de los buenos resultados, y la necesidad de una nueva infiltración, o una eventual cirugía de descompresión, ante el fracaso de la primera terapia.

Tabla III

\section{RESULTADOS DE LA INFILTRACIÓN DEL NERVIO PUDENDO POR VÍA TRANSGLÚTEA}

\begin{tabular}{ccccc}
\hline Paciente & $\begin{array}{c}\text { Dolor } \\
\text { previo }\end{array}$ & $\begin{array}{c}\text { Dolor } \\
\text { posterior }\end{array}$ & $\begin{array}{c}\text { Tiempo } \\
\text { procedimiento }\end{array}$ & Complicaciones \\
\hline 1 & 8 & 5 & 30 min & no \\
2 & 7 & 4 & 20 min & no \\
3 & 7 & 3 & 25 min & no \\
4 & 8 & 2 & 20 min & no \\
5 & 8 & 4 & 22 min & no \\
\hline
\end{tabular}




\section{BIBLIOGRAFÍA}

1. Amarenco G, Lanoe $Y$, Perrigot M, Gordal H. A new canal syndrome: compression of the pudendal nerve in Alcock's canal or perineal paralysis of cyclists. Presse Med 1987;16(8):399.

2. Lema R, Ricci P. Atrapamiento del nervio pudendo: un sindrome por conocer. Rev Chil Obstet Ginecol 2006;71(3):207-15.

3. Amarenco G, Lanoe Y, Ghnassia RT, Goudal H, Perrigot M. Alcock's canal syndrome and perineal neuralgia. Rev Neurol (Paris) 1988; 144(8-9):523-6.

4. Beco J, Climov D, Bex M. Pudendal nerve decompression in perineology: a case series. BMC Surg 2004;4:15.

5. Benson JT, Griffis K. Pudendal neuralgia, a severe pain syndrome. Am J Obstet Gynecol 2005 ;192(5):1663-8

6. Bautrant E, de Bisschop E, Vaini-Elies V, et al. Modern algorithm for treating pudendal neuralgia: $212 \mathrm{ca}-$ ses and 104 decompressions. J Gynecol Obstet Biol Reprod (Paris) 2003;32(8 Pt 1):705-12.

7. Amarenco G, Savatovsky I, Budet C, Perrigot M. Perineal neuralgia and Alcock's canal syndrome. Ann Urol (Paris) 1989;23(6):488-92.

8. Amarenco G, Le Cocquen-Amarenco A, et al. Perineal neuralgia. Presse Med 1991;20(2):71-4.

9. Le Tallec de Certaines $\mathrm{H}$, Veillard D, Dugast $\mathrm{J}$, et al. Comparison between the terminal motor pudendal nerve terminal motor latency, the localization of the perineal neuralgia and the result of infiltrations. Analysis of
53 patients. Ann Readapt Med Phys 2007;50(2):65-9.

10. Galaup JP. Dyschezia, pudendal neuropathy, and functional pelvico-perineal disorders. Prog Urol 2000;10(1):114-22.

11. Antolak SJ Jr, Hough DM, Pawlina W, Spinner RJ. Anatomical basis of chronic pelvic pain syndrome: the ischial spine and pudendal nerve entrapment. Med Hypotheses 2002;59(3):349-53.

12. Robert R, Bensignor M, Labat JJ, et al. Perineal pain. Neurochirurgie 2004;50(5):533-9.

13. Schraffordt SE, Tjandra JJ, Eizenberg N, Dwyer PL. Anatomy of the pudendal nerve and its terminal branches: a cadaver study. ANZ J Surg 2004;74(1-2):236.

14. Robert R, Prat-Pradal D, Labat JJ, et al. Anatomic basis of chronic perineal pain: role of the pudendal nerve. Surg Radiol Anat 1998;20(2):93-8.

15. Amarenco G, Denys P, Kerdraon J. Neuropathy due to stretching of the internal pudendal nerve and female urinary incontinence. J Urol (Paris) 1992;98(4):196-8.

16. Shafik A. Endoscopic pudendal canal decompression for the treatment of fecal incontinence due to pudendal canal syndrome. J Laparoendosc Adv Surg Tech A 1997;7(4):227-34.

17. Robert R, Labat JJ, Bensignor M, Glemain P, Deschamps C, Raoul S, Hamel O. Decompression and transposition of the pudendal nerve in pudendal neuralgia: a randomized controlled trial and long-term evaluation. Eur Urol 2005;47(3):403-8. 